\title{
Characterisation of the Trypanosoma brucei rhodesiense isolates from Tanzania using serum resistance associated gene as molecular marker
}

\author{
S.N. KIBONA ${ }^{1 *}$, K. PICOZZI ${ }^{2}$, L. MATEMBA ${ }^{1}$ and G.W. LUBEGA ${ }^{3}$ \\ ${ }^{1}$ National Institute for Medical Research, P.O Box 482, Tabora, Tanzania \\ ${ }^{2}$ Centre for Tropical Veterinary Medicine, University of Edinburgh, Roslin, UK \\ ${ }^{3}$ Faculty of Veterinary Medicine, Makerere University, Kampala, Uganda
}

\begin{abstract}
Serum resistance associated (SRA) gene has been found to confer resistance to the innate trypanolytic factor (TLF) found in normal human serum; thus allowing Trypanosoma brucei brucei to survive exposure to normal human serum. This study was carried out to examine the presence of SRA gene and identify the origin of $T$. $b$. rhodesiense isolates from three districts in Tanzania, namely Kibondo, Kasulu and Urambo. Twenty-six T. b. rhodesiense isolates and two references $T$. $b$. rhodesiense isolates from Kenya were examined for SRA gene using simple Polymerase Chain Reaction technique. The gene was found to be present in all 26 T. b. rhodesiense isolates including the two references isolates from Kenya. The SRA gene was confirmed to be specific to T. $b$. rhodesiense since it could not be amplified from all other Trypanozoon including T. b. gambiense; and gave an amplified fragment of the expected size (3.9kb), confirming that all these isolates were T. b. rhodesiense of the northern variant. Although the geographic distributions of T. b. gambiense and T. b. rhodesiense are clearly localized to west/central Africa and eastern Africa, respectively, natural movement of people and recent influx of large number of refugees into Tanzania from the Democratic Republic of Congo, could have brought T. b. gambiense in western Tanzania. The overlap in distribution of both of these pathogenic sub-species could result in erroneous diagnoses since both trypanosome sub-species are morphologically identical, and currently serologic methods have low specificity. Both the susceptible and resistant T.b. rhodesiense isolates possessed the SRA gene suggesting that there is no correlation between drug resistance and presence of SRA gene. The use of SRA gene helps to confirm the identity and diversity of some of the isolates resistant to various drugs.
\end{abstract}

Keywords: Trypanosoma brucei rhodesiense, resistance, SRA gene, PCR, Tanzania

\section{Introduction}

Innate protective molecules in the blood of primates influence the host range of African trypanosomes. Human blood, unlike the blood of other mammals, has efficient trypanolytic activity, and this needs to be counteracted by these parasites. Trypanosoma brucei consists of three sub-species, non-human infective Trypanosoma brucei brucei and human infective $T . b$. rhodesiense and T. $b$. gambiense that are indistinguishable by conventional morphological, biochemical and antigenic criteria but differ by their geographical distribution, host specificity (Mehlitz et al., 1982; Noireau et al., 1989; Hide et al., 1994; Gibson, 2001). A sub-fraction of human high-density lipoprotein (HDL) containing apolipoprotein A-I, apolipoprotein L-I, and haptoglobin-related protein is toxic to T. b. brucei but not T. b. rhodesiense (Pays et al., 2006).

This question of sub-speciation has been resolved in the past, by inoculating human volunteers with trypanosomes isolated from other animals (Heisch et al., 1958; Onyango et al., 1966), or more acceptably by in vitro tests involving incubation of trypanosomes with human blood in the blood incubation test (BIIT) (Rickman \& Robson, 1970) or with serum in human serum resistance test (HSRT) (Brun \& Jenni, 1987). Biochemical and molecular characterization such as isoenzyme electrophoresis, restriction fragment length polymorphisms using ribosomal DNAs (RFLP) have shown significant differences between the two subspecies, but have not defined clearly the criteria for identifying the human-infective sub-species isolated from animal reservoirs or vectors. However, these methods are time consuming, laborious and inappropriate for the field situation (Gibson, 1989; Hide et al., 1990, 1994, 1998). Therefore, the search for techniques to distinguish $T$. $b$. rhodesiense from T. $b$. brucei and hence its human infectivity is important.

T. b. brucei causes nagana in cattle but is not pathogenic in humans because this sub-species is lyzed by high-density lipoproteins (HDL) present in human serum. It is thought that $T$. $b$. rhodesiense evolved from a $T$. $b$. brucei-like ancestor and expresses a defence protein that ablates the anti-trypanosomal activity of human HDL. The ability of T. $b$. rhodesiense and T. $b$. gambiense to be resistant to normal human serum (NHS) enabled them to parasitize humans and cause sleeping sickness. The mechanism of resistance to NHS is still a subject for debate but previously it was believed to be due to defect in the uptake of HDL factor (Hager \& Hajduk, 1997). 
This search has been nearly concluded by the discovery of the mechanism of human serum resistance in $T$. $b$. rhodesiense. A single gene, known as serum resistance associated (SRA) gene has been found to confer resistance to the innate trypanolytic factor (TLF) found in normal human serum. This gene allows T.b brucei to survive the exposure to normal human serum (Xong et al., 1998). In long, slender bloodstream forms of $T$. b. rhodesiense, the expression of SRA allows neutralization of APOL1 in the lysosome. This mechanism of resistance considerably differs from the previously proposed mechanism, the selective inhibition of endocytosis of the trypanolytic factor.

The SRA gene was isolated for the first time from T. b. rhodesiense isolate from Uganda (De Geef et al., 1989). The SRA gene is transcribed from one of the multiple telomeric loci where variant surface glycoprotein (VSG) genes are expressed (VSG expression sites). This expression site is selected when trypanosomes are grown in presence of NHS (Van Xong et al., 1998) The product of SRA is a atypical VSG of shorter than average length being 410 amino acids instead of approximately 490 (Vanhamme et al., 2003).

The human SRA gene has been found in all T. $b$. rhodesiense isolates examined from sleeping sickness foci throughout East Africa, but not in T. b. brucei or any other trypanosomes of subgenus Trypanozoon, including T. b. gambiense (De Greef et al., 1992; Welburn et al., 2001; Radwanska et al., 2002; Gibson et al., 2002). This suggests that T. b. gambiense resists lysis through a different mechanism. Indeed $T . b$. gambiense and T. b. rhodesiense appear to differ in their mechanism of resistance to normal human serum. In contrast to $T . b$. brucei, the subspecies $T . b$. gambiense and T. b. rhodesiense escape the trypanolytic activity of human serum and cause sleeping sickness pathology. T. b. gambiense is permanently resistant to human serum whereas $T$. $b$. rhodesiense loses resistance after being isolated from humans and transferred to other animals (Hawking, 1977).

Recent advances in this field of research include a breakthrough in the diagnosis of sleeping sickness, for which the presence of SRA has proved to be a reliable marker of infection with $T$. $b$. rhodesiense (Gibson 2005; Gibson et al, 2002; Welburn et al., 2001). This marker can also be used to distinguish $T$. b. rhodesiense from T. b. gambiense.

In this study, the SRA characterization was undertaken because the study area in western Tanzania is home to thousands of refugees from the highly T. $b$. gambiense endemic country, the Democratic Republic of Congo. It was thought that natural movement of people and influx of large number of refugees could have brought $T$. b. gambiense into western Tanzania, and also some of the isolates, both drug resistant and sensitive, identified probably could be cases of T. $b$. gambiense and not T. rhodesiense. The SRA marker was also used to characterize geographical origin of the isolates because this has migration implications that influence spread of drug resistant strains in the region.

\section{Materials and Methods}

\section{Study areas}

The study area included Kibondo, Kasulu and Urambo Districts in western Tanzania. The study area has been described in detail by Malele et al. (2006). Purposeful sampling method was used to select Kibondo, Kasulu and Urambo, because of high human African trypanosomiasis reporting cases for the past five years (Ministry of Health, unpubl.).

\section{Isolation of trypanosomes}

During the survey, people suspected to be infected with trypanosomes based on clinical observations were examined by blood smear and haematocrit centrifugation technique (HCT) in order to confirm the infection. Blood samples $(2 \mathrm{ml})$ were collected from confirmed sleeping sickness cases by venipuncture under the supervision of a medical doctor. Then, the patients were referred to hospital for treatment according to the stage of their illness. Each blood sample collected was divided into two portions, which were cryopreserved in liquid nitrogen. One portion was used for propagation of the isolates to the mice and the other was kept for future references.

\section{DNA extraction from $T . \quad b$. rhodesiense isolates}

Genomic DNA was extracted using a commercial kit (Puregene DNA isolation kit D-7000A, Gentra Systems, Minneapolis, USA) following the manufacturer's instructions with minor modifications. A total of $500 \mathrm{ml}$ of blood was mixed with $1500 \mathrm{ml}$ of $\mathrm{RBC}$ lysis solution and incubated at room temperature for 5 minutes. The mixture was then span at $13000 \mathrm{~g}$ for 2 minutes and the supernatant discarded. The pellet was resuspended in about $50 \mathrm{ml}$ of residual fluid, which was mixed with $250 \mathrm{ml}$ cell lysis solution containing proteinase $\mathrm{K}(100 \mathrm{mg} / \mathrm{ml})$, and incubated at $55^{\circ} \mathrm{C}$ for 1 hour. RNAse $(29 \mathrm{mg} / \mathrm{ml})$ was then added and the mixture incubated at $37^{\circ} \mathrm{C}$ for another 45 minutes. 
Protein was then isolated by addition of $200 \mathrm{ml}$ of protein precipitation solution, and incubated on ice for 5 minutes, followed by micro-centrifugation for 5 minutes at maximum speed. From that supernatant, DNA was precipitated by addition of $600 \mathrm{ml}$ isopropanol, and the pellet was washed with $70 \%$ ethanol. The dry pellet was finally dissolved in $20 \mathrm{ml}$ of the DNA hydration solution included in the kit and allowed to rehydrate at $65^{\circ} \mathrm{C}$ for 1 hour.

\section{PCR analysis for T. brucei subgroup}

Primers used to amplify species specific DNA targets for $T$. brucei subgroup from isolates were TBR 1 and 2 with sequence TBR 15 '-CGAATG AAT AAT AAA CAA TGC GCA GT-3' and TBR2 5'-AGAACC ATT TAT TAG CTT TGT TGC-3' (Artama et al., 1992). The concentration of the primers was $0.4 \mu \mathrm{M}$. Standard PCR amplifications were carried out in $25 \mu 1$ reactions mixtures containing the final concentrations, $10 \mathrm{mM}$ TrisHCL pH 8.3, $50 \mathrm{mM} \mathrm{KCl}, 1.5 \mathrm{mM} \mathrm{MgCl}, 200$ $\mu \mathrm{m}$ of each of the 4 deoxynucleoside triphosphates and 1 unit of RED Taq DNA polymerase (Sigma). The amplification conditions were $\left(30\right.$ cycles) $94^{\circ} \mathrm{C}$ for $30 \mathrm{sec}, 60^{\circ} \mathrm{C}$ for $60 \mathrm{sec}$ and $72^{\circ} \mathrm{C}$ for $30 \mathrm{sec}$. PCR products were separated by electrophoresis in $1.5 \%$ (w/v) agarose gel containing $0.5 \mu \mathrm{g} / \mathrm{ml}$ ethidium bromide and visualized under ultraviolet light.

\section{SRA amplification from genomic DNA}

PCR primers for SRA amplification were based on the DNA sequence of the SRA gene and its homologues to Kenyan T. b. rhodesiense isolates (EMBL accession number AF097331). Primers B537 (forward) (5'-CCA TGG CCT TTG ACG AAG AGC CCG-3') and B538 (reverse) (5'-CTC GAG TTT GCT TTT CTG TAT TTT TCC T) at 2' $\mu \mathrm{M}$ were complimentary to the 5' and 3' ends of the published SRA gene (accession no. AF097331). These primers were used to screen all trypanosome genomic DNA from 28 isolates for presence of SRA fragment by PCR (Welburn et al., 2001). The amplification condition was 30 cycles of $94^{\circ} \mathrm{C}$ for $45 \mathrm{sec}, 55^{\circ} \mathrm{C}$ for $45 \mathrm{sec}$ and $72^{\circ} \mathrm{C}$ for $90 \mathrm{sec}$. Reaction volumes $(25 \mu \mathrm{l})$ which contained 1 U HotStarTaq DNA polymerase (Qiagen) supplemented with 1 U Pfu polymerase (Promega) and $4 \mathrm{mmol} / \mathrm{L} \mathrm{MgCl}_{2}$ were used. In each experiment positive controls for T. b. brucei, T. b. gambiense and $T b$. rhodesiense were used.

\section{Screening for northern and southern SRA gene variants}

The primers were used to screen trypanosome genomic DNA in order to identify the category (northern or southern) of SRA gene of the isolates; forward SRA H: (5'-GTACCTTGGCGCGCTCC CTGG-3') and reverse SRAJ :(5'-GTA CCT TGGCGCGCT CGCGCTG-3') (Gibson et al., 2002). PCR conditions for this amplification were denaturations at $95^{\circ} \mathrm{C}$ for $30 \mathrm{sec}$, annealing at $60^{\circ} \mathrm{C}$ for $30 \mathrm{sec}$ and extension at $72^{\circ} \mathrm{C}$ for $60 \mathrm{sec}$.

\section{Ethics declaration}

This study was approved by Medical Research Coordination Committee of the National Institute for Medical Research, Tanzania. The free and informed consent of all patients who participated in this study or their legal guardians was obtained before commencement of the study

\section{Results}

\section{Confirmation of $\mathrm{T}$. brucei subgroup}

All isolates were first confirmed as T. brucei subgroup by PCR analysis (Figure 1) using Trypanozoon primers TBR1 and TBR2 before screening them for the presence of SRA gene. Since the expected band of size of $177 \mathrm{bp}$ was present in all, isolates screened were confirmed to belong to T. brucei subgroup.

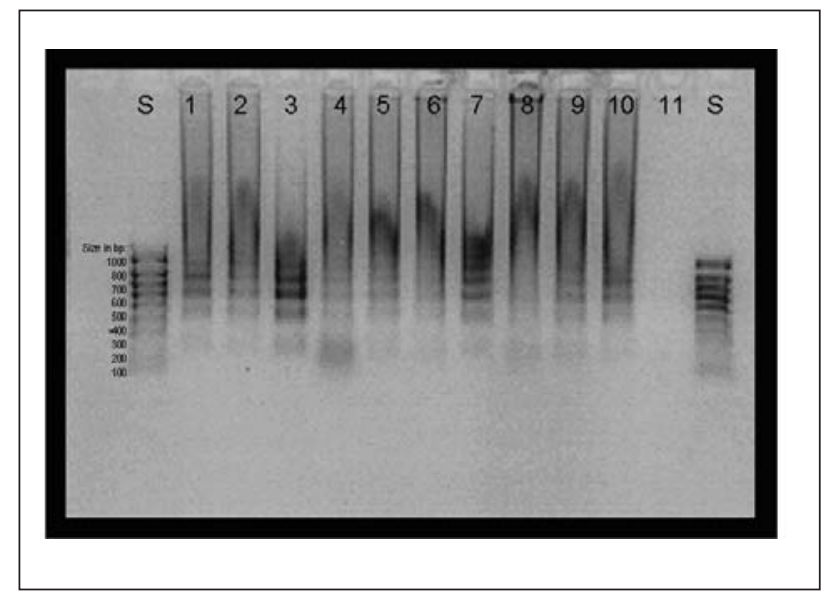

Figure 1: PCR for the confirmation of T. brucei subgroup of the isolates studied. Representative samples 1-10 were screened by PCR using Trypanozoon primers, TBR1 and TBR2. Lane 11 corresponds to a negative control. Lane S contains the 100 bp marker (BiolineUK). Lane 1-10 represents isolates TMRS 3(9), TMRS 10(6), TMRS 11(2), TMRS 12 (2), TMRS 13 (2), TMRS 4 (1), TMRS 1 (13), TMRS 2 (11), KETRI 1989 and KETRI 2653, respectively. The presence of expected PCR product s of $177 \mathrm{bp}$ or multiples thereof confirms the isolates as T. brucei subgroup 


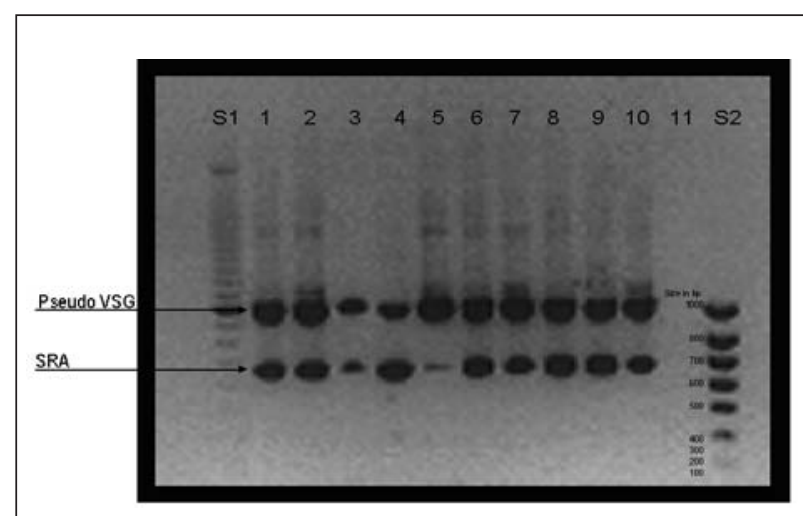

Figure 2: PCR for the identification of SRA gene among the isolates studied. Isolates were screened by PCR using primers B537 and B538 to amplify SRA gene. Lane S1 contains Hyperladder 1. Only representative isolates are shown in Lane 1-10, namely, TMRS 3(9), TMRS 10(6), TMRS 11(2), TMRS 12(4), TMRS 13(2), TMRS 4(1), TMRS 1(13), TMRS 2(11), KETRI 1989 and KETRI 2653 respectively. Lane 11 contains negative control and Lane S2 contains $1000 \mathrm{bp}$ marker. The presence of expected PCR product of $670 \mathrm{bp}$ or multiples thereof confirms the presence of SRA gene.

\section{Amplification of SRA gene}

The SRA fragment of approximately $670 \mathrm{bp}$ (Figure 2) was amplified from all $26 T$. b. rhodesiense isolates including 2 reference isolates from Kenya. Thus the SRA gene was present in both drug susceptible and drug-resistant T. b. rhodesiense isolates (Table 1).

\section{Screening for the northern and southern SRA gene variants}

To determine whether they belong to northern or southern origin these isolates were screened by PCR using SRA primers (SRA H and SRA J). A prominent band of $3.9 \mathrm{~kb}$, was amplified from all T. b. rhodesiense isolates from Tanzania except two isolates probably due to technical error. The $3.9 \mathrm{~kb}$ band was also amplified from reference strains KETRI 1989 and KETRI 2356 from Kenya. No band of 3.5kb, which is a marker for southern variant, was amplified from these isolates. All the 24 isolates from Tanzania had the northern variant of the SRA gene (Table 1). The SRA gene was specific for $T$. $b$. rhodesiense and could not be amplified from T.b. gambiense and T. b. brucei.

Table 1: Human SRA gene amplification from T. b. rhodesiense genomic DNA

\begin{tabular}{|c|c|c|c|c|c|}
\hline Isolate & Origin & Year & Drug sensitivity status* & SRA** & Category*** \\
\hline TMRS $10(6)$ & Kasulu & 1999 & $\mathrm{R}$ IS $(1 \mathrm{mg})$ & + & $\mathrm{N}$ \\
\hline TMRS $11(2)$ & Kasulu & 2000 & $\mathrm{R} M(5 \mathrm{mg}) ; \mathrm{D}(14 \mathrm{mg})$ & + & $\mathrm{BA}$ \\
\hline TMRS $12(4)$ & Kasulu & 1999 & $\mathrm{R} \mathrm{M}(5$ and $10 \mathrm{mg}) ; \mathrm{D}(14 \mathrm{and} 28 \mathrm{mg})$ & + & $\mathrm{N}$ \\
\hline TMRS $13(2)$ & Kasulu & 1999 & $S$ & + & $\mathrm{N}$ \\
\hline TMRS 15 (6) & Kibondo & 1999 & $\mathrm{~S}$ & + & $\mathrm{N}$ \\
\hline TMRS 7 (2) & Kibondo & 1999 & $S$ & + & $\mathrm{N}$ \\
\hline TMRS 3 (2) & Kibondo & 1999 & $\mathrm{~S}$ & + & $\mathrm{N}$ \\
\hline TMRS $3(3)$ & Kibondo & 1999 & S & + & $\mathrm{N}$ \\
\hline TMRS 3 (11) & Kibondo & 2002 & R M (5mg) & + & $\mathrm{N}$ \\
\hline TMRS 3 (6) & Kibondo & 2002 & $\mathrm{~S}$ & + & $\mathrm{N}$ \\
\hline TMRS 4 (1) & Urambo & 2002 & R $\mathrm{SU} \quad(5 \mathrm{mg})$ & + & $\mathrm{N}$ \\
\hline TMRS 2 (2) & Urambo & 2002 & $\mathrm{~S}$ & + & $\mathrm{N}$ \\
\hline TMRS 1 (13) & Urambo & 2002 & $\mathrm{R} \quad$ IS (1mg) & + & $\mathrm{N}$ \\
\hline TMRS 5 (1) & Tabora & 2000 & $\mathrm{~S}$ & + & $\mathrm{N}$ \\
\hline TMRS $3(7)$ & Kibondo & 2000 & $\mathrm{~S}$ & + & $\mathrm{N}$ \\
\hline TMRS 9 (5) & Kasulu & 2002 & $\mathrm{~S}$ & + & $\mathrm{N}$ \\
\hline TMRS 2(11) & Urambo & 2002 & R SU (5mg); IS (1mg) & + & $\mathrm{N}$ \\
\hline TMRS $8(13)$ & Kasulu & 2001 & $S$ & + & $\mathrm{N}$ \\
\hline TMRS $3(9)$ & Kibondo & 2000 & $S$ & + & $\mathrm{BA}$ \\
\hline TMRS $10(3)$ & Kasulu & 2000 & $\mathrm{~S}$ & + & $\mathrm{N}$ \\
\hline TMRS 10 (4) & Kasulu & 2000 & $S$ & + & $\mathrm{N}$ \\
\hline TMRS $11(3)$ & Kasulu & 2000 & $\mathrm{~S}$ & + & $\mathrm{N}$ \\
\hline TMRS $12(2)$ & Kasulu & 2000 & $S$ & + & $\mathrm{N}$ \\
\hline TMRS 1 (2) & Urambo & 2001 & $S$ & + & $\mathrm{N}$ \\
\hline TMRS 3 (12) & Kibondo & 2002 & $S$ & + & $\mathrm{N}$ \\
\hline TMRS $12(3)$ & Kasulu & 1999 & $S$ & + & $\mathrm{N}$ \\
\hline KETRI 1989 & Kenya & 1989 & $\mathrm{R} S \mathrm{SU}$ and $\mathrm{D}$ & + & $\mathrm{N}$ \\
\hline KETRI 2356 & Kenya & 1997 & $\mathrm{R} \quad \mathrm{M}$ & + & $\mathrm{N}$ \\
\hline
\end{tabular}

*Drug sensitivity status is given as resistant (R) or sensitive (S).

$\mathrm{M}=$ Melarsoprol, $\mathrm{D}=$ Diminazene, $\mathrm{SU}=$ Suramin, $\mathrm{IS}=$ Isometamidium; BA= Expected but not amplified

Dosage of drug at which the isolate is resistant is given in brackets.

${ }^{* *}$ The presence of SRA gene is indicated by + signs; ${ }^{* *}$ All isolates were of the Northern $(\mathrm{N})$ variant except two isolates which could not be classified since no band was amplified from them. 


\section{Discussion}

Several studies have shown that $T . b$. rhodesiense and T. b. gambiense are able to infect humans due to their resistance to the cytotoxic action of normal human serum. A subfraction of human high-density lipoprotein (HDL) containing apolipoprotein A-I, apolipoprotein L-I, and haptoglobin-related protein is toxic to $T$. $b$. brucei but not the human sleeping sickness parasite $T . b$. rhodesiense and T. b. gambiense (Faulkner et al., 2006; Pays et al., 2006). These two sub-species are largely indistinguishable from $T$. $b$. brucei except for their resistance to the cytotoxic action of human serum and their ability to infect humans. Therefore, the defining phenotypic trait of human sleeping sickness trypanosomes is their resistance to TLF-mediated lysis. The human serum resistance associated (SRA) gene first isolated from a Ugandan strain of T. b. rhodesiense has been shown to be capable by itself of conferring resistance to antitrypanosomal activity of human HDL and the trait of human infectivity on T.b. brucei by transfection (Gibson et al., 2001).

To directly investigate this possibility, a study was carried out elsewhere for in vitro selection to generate human HDL-resistant T. b. brucei. The results showed that conversion of $T$. $b$. brucei from human HDL sensitive to resistant correlates with changes in the expression of the variant surface glycoprotein (VSG) and abolished uptake of the cytotoxic human HDLs (Faulkner et al., 2006). These findings demonstrate that resistance to human HDLs can be acquired by $T$. b. brucei. Furthermore T. b. rhodesiense is likely to have arisen as a clone of $T . b$. brucei that differs mainly or solely by its ability to express SRA on selection in human serum. This gene has also been identified in several other isolates of T.b. rhodesiense, but not in $T$. b. brucei or any other trypanosomes of subgenus Trypanozoon, including another human infective $T$. b. gambiense. This gene has been found in all T. $b$. rhodesiense isolates examined from sleeping sickness foci in Tanzania confirming that it is found throughout East Africa (Ethiopia, Uganda, Kenya, Rwanda, and Zambia).

The 26 stocks of $T$. b. rhodesiense from patients in 3 districts of Tanzania were examined for the presence of the SRA gene and gave an amplified fragment of the expected size confirming that all these isolates were T. b. rhodesiense. Although the geographic distributions of T. $b$. gambiense and T. $b$. rhodesiense are clearly localized to west/central Africa and eastern Africa, respectively, natural movement of people and recent influx of large number of refugees into Tanzania from the Democratic Republic of Congo, could have brought $T . b$. gambiense in western Tanzania. In the Democratic Republic of Congo, the reported prevalence of $T$. b. gambiense sleeping sickness is up to $80 \%$ in some foci (Van Nieuwenhove et al., 2001). The overlap in distribution of both of these pathogenic sub-species can result in erroneous diagnoses since both trypanosome sub-species are morphologically identical, and the currently available serologic techniques have low specificity (Radwanska et al., 2002). Nonetheless, since these subspecies show differential drug sensitivities, a correct differential diagnosis between $T$. b. gambiense and $T$. $b$. rhodesiense is essential for unambiguous diagnosis of drug resistance. Therefore, the development of a simple molecular technique such as the SRA genebased PCR may be essential for the correct diagnosis of resistance attributable to $T$. $b$. rhodesiense.

Both the susceptible and resistant T. b. rhodesiense isolates possessed the SRA gene suggesting that there is no correlation between drug resistance and presence of SRA gene. The lack of this kind of correlation suggests that these two phenomena arose by independent mechanisms. The drug resistance is most likely to be due to selection by drug pressure (Kibona et al., 2006) and this selection does not affect the SRA gene. It is a valid objective to try to link observed differences in drug sensitivity with genetic markers such as the serum resistance. However, there have been few studies attempting to link drug resistance with other genotypes in East Africa. Matovu et al. (1997) carried out in vitro screening of the Ugandan T. $b$. gambiense and T. b. rhodesiense isolates, identified by human serum resistance (HSR) for susceptibility to melarsoprol, diminazene and isometamidium in vitro and found one $T$. $b$ rhodesiense isolate resistant to the tested drugs. In view of that, the potential association between genetic markers and other characteristics such drug resistance or pathogenicity are areas worth to be explored.

The SRA gene cannot be used as sole marker for human infectivity in T. brucei sub-species because, it is absent in T. gambiense which is human infective. However, since a large variety of wild and domestic animals serve as reservoirs for both subspecies, SRA gene is a useful marker for the identification of trypanosomes of the T. b. rhodesiense subspecies that can infect humans (Gibson, 2001). Once the human infectivity of an isolate has been established then SRA gene can be used to distinguish T. b. rhodesiense from T. b. gambiense.

Although the $S R A$ gene is conserved among T. $b$. rhodesiense isolates, there are two major sequence variants, designated northern and southern to reflect 
geographical origin (Gibson et al., 2002). This division tallies with previous clinical and molecular characterization studies indicating the existence of northern and southern strains of $T$. $b$. rhodesiense (Hide \& Tilley, 2001; Ormerod, 1967; Gibson et al., 1980; Macleod et al., 2000). In this study a prominent band of a $3.9 \mathrm{~kb}$ was amplified from Tanzanian isolates of $T$. $b$. rhodesiense genomic DNA. Similar findings have been observed in isolates from Uganda and Kenya (Gibson et al. 2002; Gibson \& Ferris, 2003), meaning that all these isolates had the northern variant of the SRA gene. Thus the northern variant seems to cover the entire East Africa region including Tanzania, Kenya and Uganda.

Nevertheless, since the SRA gene resembles VSG genes and the extent of genetic evolution of this gene is currently unknown, one cannot exclude the existence of $T$. $b$. rhodesiense parasites with defective SRA variants or the existence of parasites with a modified SRA gene. Only two SRA genes have been characterized that differ from each other by a few point mutations (De Greef\& Hamers, 1994). Moreover, in all cases where this was studied, the SRA gene appears to be a member of a large gene family that contains many pseudo genes; thus, the possibility exists that none of these sequences are functional (De Greef \& Hamers, 1994). Recently, Radwanska et al. (2002) observed an interesting finding with strain TREU927/ 4 , which is currently used as a reference $T$. brucei strain for genome sequencing. This strain was found to be resistant to lysis by NHS without expression of the SRA gene, even though SRA gene-related sequences were present.

Future studies involving large collections of field isolates are needed to confirm the reliability of the SRA gene PCR in identifying T. $b$. rhodesiense as the causative agent of human trypanosomiasis in different parasite foci.

\section{Acknowledgements}

This study was financially supported by UNDP/World Bank/WHO Special Programme for Research and Training in Tropical Diseases (Project ID A00304 linked to ID 971013). All the laboratory work was carried out at Faculty of Veterinary Medicine, Makerere University, Kampala, Uganda. We are grateful to Kenya Trypanosomiasis Research Institute, Kikuyu, Kenya for providing the T. $b$. rhodesiense isolates which were used as controls.

\section{References}

Artama, W.T., Agey, M.W. \& Donelson, J.E. (1992) DNA comparisons of Trypanosoma evansi (Indonesia) and Trypanosoma brucei spp. Parasitology 104, 67-74
Brun, R. \& Jenni, L. (1987) Human serum resistance of metacyclic forms of Trypanosoma b. brucei, $T$. b. rhodesiense and T. b. gambiense. Parasitology Research 73, 218-223.

De Greef, C. \& Hamers, R. (1994) The serum resistance-associated (SRA) gene of Trypanosoma brucei rhodesiense encodes variant surface glycoprotein-like protein. Molecular \& Biochemical Parasitology 68, 277-284.

De Greef, C., Chimfwembe, E., Kihang'a Wabacha, J., Bajyana Songa, E. \& Hamers, R.(1992) Only the serum-resistant bloodstream forms of'Trypanosoma brucei rhodesiense express the serum resistance associated (SRA) protein. Annales de la Societe Belge de Medicin Tropicale 72,13-21.

Faulkner S.D., Oli, M.W., Kieft, R., Cotlin, L., Widener, J., Shiflett, A., Cipriano, M.J., Pacocha, S.E., Birkeland, S.R., Hajduk, S.L. \& McArthur, A.G. (2006) In vitro generation of human highdensity-lipoprotein-resistant Trypanosoma brucei brucei. Eukaryotic Cell 5, 1276-1286.

Gibson, W. (2001) Molecular characterization of field isolates of human pathogenic trypanosomes. Tropical Medicine and International Health 6, 401-406.

Gibson, W.C. (1989) Analysis of a genetic cross between Trypanosoma brucei rhodesiense and T. b. brucei. Parasitology 99, 391-402.

Gibson, W.C. (2005) The-SRA gene: the key to understanding the nature of Trypanosoma bruce $i$ rhodesiense. Parasitology 131, 143-150.

Gibson, W., Backhouse, T. \& Griffiths, A. (2002) The human serum resistance associated gene is ubiquitous and conserved in-Trypanosoma brucei rhodesiense throughout East Africa. Infection and Genetic Evolution 1, 207-214.

Gibson, W.C, Marshall, T.C. \& Godfrey, D.G (1980) Numerical analysis of enzyme polymorphism: a new approach to the epidemiology and taxonomy of trypanosomes of the subgenus Trypanozoon. Advanced Parasitology 18, 175-246.

Gibson, W. \& Ferris, V. (2003) Conservation of the genomic location of the human serum resistance associated gene in Trypanosoma b. rhodesiense. Molecular \& Biochemical Parasitology 130, 159-162.

Gibson, W.C. \& Mizen V.H. (1997) Heritability of the trait for human infectivity in genetic crosses of Trypanosoma brucei sp. Transactions of the Royal Society of Tropical Medicine and Hygiene 91, 236-237.

Hager, K.M. \& Hajduk, S.L. (1997) Mechanism of resistance of African trypanosomes to cytotoxic human HDL. Nature 385, 823-825. 
Hawking, F. (1977) The resistance to human plasma of Trypanosoma brucei, $T$. rhodesiense and $T$. gambiense: analysis of the composition of trypanosome strains. Transactions of the Royal Society of Tropical Medicine and Hygiene $\mathbf{7 0 ,}$ 504-512.

Hide, G. \& Tilley, A. (2001) Use of mobile genetic elements as a tool for molecular epidemiology. International Journal of Parasitology 31, 599602.

Hide, G., Angus, S.D., Holmes, P.H., Maudlin, I. \& Welburn, S.C. (1998) Trypanosoma brucei: comparison of circulating strains in an endemic and an epidemic area of a sleeping sickness focus. Experimental Parasitology 89, 21-29.

Hide, G., Cattand, P., Le Ray, D., Barry, D.J. \& Tait, A. (1990) The identification of Trypanosoma brucei subspecies using repetitive DNA sequences. Molecular and Biochemical Parasitology 39, 213-226.

Hide, G., Welburn, S., Tait, A. \& Maudlin, I. (1994) Epidemiological relationship of T. brucei stocks from southeast Uganda: evidence for different population structures in human infective and non-infective isolates. Parasitology 109, 95-111.

Kibona, S.N., Matemba, L., Kaboya, J.S. \& Lubega, G.W. (2006) Drug-resistance of Trypanosoma b. rhodesiense isolates from Tanzania. Tropical Medicine and International Health 11, 44-155.

MacLeod, A., Tweedie, A., Welburn, S.C., Maudlin, I., Turner, C.M.R. \& Tait, A. (2000) Minisatellite marker analysis of Trypanosoma brucei: reconciliation of clonal, panmictic, and epidemic population genetic structures. Proceedings of the National Academy of Science USA. 97, 1344213447.

Malele, I.I., Kibona, S.N., Matemba, L.E., Sahani, K., Swilla, J., Mwalimu, C.D., Mayala, B.K., Kimaro, E., Msumary, C. \& Kalinga, R.B. (2006) Human African trypanosomiasis and challenges to its control in Urambo, Kasulu and Kibondo districts, western Tanzania.-Tanzania Health Research Bulletin-8, 80-85.

Matovu, E., Iten, M. \& Enyaru, J.C.K. (1997) Susceptibility of Ugandan T.b. rhodesiense isolated from man and animal reservoirs to diminazene, Isometamidium and melarsoprol. Tropical Medicine and International Health 2, 3-18.

Mehlitz, D., Zillmann, U., Scott, C.M. \& Godfrey, D.G. (1982) Epidemiological studies on the animal reservoir of gambiense sleeping sickness. III. Characterization of Trypanozoon stocks by isoenzyme and sensitivity of the human serum. Tropical Medicine and Parasitology 33, 113-118.

Noireau, F., Paindavoine, P., Lamesre, J.L., Declercq, J. \& Miaka, C. (1989) The epidemiological importance of the animal reservoir of $T . b$. gambiense in the Congo. Tropical Medicine and Parasitology 40, 9-11.

Onyango, R.J., Van Hoeve, K. \& De Raadt, P. (1966) The epidemiology of Trypanosoma rhodesiense sleeping sickness in Alego location, Central Nyanza, Kenya. I. Evidence that cattle may act as reservoir hosts of trypanosomes infective to man. Transactions of the Royal Society of Tropical Medicine and Hygiene 60, 175-182.

Ormerod, W.E. (1967) Taxonomy of the sleeping sickness trypanosomes. Journal of Parasitology 53, 824-830.

Pays, E., Vanhollebeke, B. \& Vanhamme, L. (2006) The trypanolytic factor of human serum. Nature 4, 477-486.

Radwanska, M., Chamekh, M., Vanham, L., Claes me, F., Magez, S., Magnus, E., De Baetselier, P., Buscher, P. \& Pays, E. (2002) The serum resistance-associated gene as a diagnostic 165 tool for the detection of Trypanosoma brucei rhodesiense. American Journal of Tropical Medicine and Hygiene 67, 684-690.

Rickman, L.R. \& Robson, J. (1970) The testing of proven Trypanosoma brucei and T. rhodesiense strains by the blood incubation infectivity test. Bulletin of the World Health Organization 42, 911-916.

Van Nieuwenhove, S., Betu-Ku Mesu, V. K., Diabakana, P., Declercq, J. \& Miaka, C. (2001) Sleeping sickness resurgence in the DRC: the past decade. Tropical Medicine and International Health 6, 335-341.

Vanhamme, L., Paturiaux-Hanocq, F., Poelvoorde, P., Nolan, D., Lins, L., Van den Abbeele, J., Pays, A., Tebabi, P., Xong, H., Jacquet, A., Moguilevsky, N., Dieu, M., Kane, J.P., De Baetselier, P., Brasseur, R. \& Pays, E. (2003.) Apolipoprotein L-1 is the trypanosome lytic factor of human serum. Nature 422, 83-87.

Welburn, S.C., Picozzi, K., Fevre, E.M., Coleman, P.G, Odiit, M., Carrington, M. \& Maudlin, I. (2001) Identification of human-infective trypanosomes 156 in animal reservoir of sleeping sickness in Uganda by means 157 of serum-resistance-associated (SRA) gene. Lancet 358, 2017-2019.

Xong, H.V., Vanhamme, L., Chamekh, M., Chimfwembe, C.E., Van Den Abbeele, J., Pays, A., Van Meirvenne, N., Hamers, R., De Baetselier, P. \& Pays, E. (1998) A VSG expression site-associated gene confers resistance to human serum in Trypanosoma rhodesiense. Cell 95, 839-846. 